\title{
Focused Ion Beam Applications in Biology
}

M. Marko*,**

*Wadsworth Center, Empire State Plaza, Albany, NY 12201

** College of Nanoscale Science and Engineering of the University at Albany, 251 Fuller Rd., Albany, NY 12203

This tutorial is intended for biologists interested in how focused ion beam (FIB) instruments might be used in their research. It will consist of a basic introduction to FIB technology, a description of the most relevant techniques used in biology, and a selection of application examples.

A FIB instrument is similar to an SEM, but produces a beam of positive ions (usually gallium) instead of electrons [1]. The ions, much heaver than electrons, can be focused by electrostatic lenses into a spot on the specimen that is a few nanometers in diameter. At beam currents of tens of picoamps or a few nanoamps, specimen material can be sputtered away to a depth of about 10 to $100 \mathrm{~nm}$ with each pass of the beam. This allows precision cutting and shaping of the specimen. Many modern FIB instruments include an SEM on the same vacuum chamber, the two-column "FIB-SEM". The use of SEM makes it more practical to image the specimen after cutting, without further damage.

The FIB is used to prepare cross-sections or to open "windows" in the specimen for an internal view by SEM, and for making specimens for TEM. When done properly, heterogeneous hard/soft materials can be milled with a smooth surface. Frozen biological material can also be FIB-milled and observed by SEM [2] or transferred to the TEM [3].

Use of FIB for TEM preparation takes three forms: (1) Thinning a portion of the specimen to TEM transparency (the "H-bar" method); this can be done at room temperature or under cryo conditions; (2) preparing a cylindrical specimen that is particularly suited to electron tomography [e.g. 4]; and (3) extracting a thin "lamella" from within a specimen and placing it on a TEM specimen holder (the "lift-out" method") [5].

Probably the FIB technique of most general impact in biology is "FIB tomography", which does not involve TEM. Here, specimens are prepared by embedding in resin, after osmium fixation and subsequent en-bloc heavy-metal staining [6]. After mechanical trimming to the area of interest, the surface is alternately FIB-milled and imaged by SEM. At each milling step, about 5-20 $\mathrm{nm}$ of material is removed, and then SEM an image is recorded using backscattered electron imaging. The $x-y$ resolution can be $5 \mathrm{~nm}$ or greater. This iterative process can proceed under software control, and it can run for days, yielding a relatively highresolution $3-\mathrm{D}$ reconstruction over a large area $[7,8,9]$.

All of the above techniques have been used extensively for over a decade in materials science, and almost as long, although with far less frequency, in biology. The first application of FIB in biology was in 1993, in which FIB was used to cut into a mite in order to image internal features [10]. Dental research represents the single largest number of publications so far [e.g. 
$11,12]$, in which cross-sections of teeth and their interface with dental implants are of particular interest. These studies include elemental analysis [13] and preparation of samples for TEM. Study of interfaces between implants and other biological material (cells, tissue, bone) make up another major category [e.g 14, 15, 16, 17], and the effect of nanoparticles on cells is also being studied [18]. Examples of the wide variety of biological specimens studied with the aid of FIB include yeast [4], chromosomes [19], atherosclerotic tissue [20], brain [7], and other organs [21].

A cutting edge use of FIB in EM structural biology is the milling of vitreously frozen specimens for TEM cryo-tomography, and some of our own work in this area [22] will be briefly presented.

References

[1] L. Giannuzzi and F. Stevie, Introduction to Focused Ion Beams, Springer, New York, 2005.

[2] M. Hayles et al., J. Microsc. 226 (2007) 263.

[3] M. Marko et al., Nat Meth. 4 (2007) 215.

[4] T. Kamino et al., J. Electron. Microsc. 53 (2004) 536.

[5] J. Heymann et al., J. Struct. Biol. 155 (2006) 63.

[6] V. Leser et al. J. Microsc. 233 (2009) 309.

[7] G. Knott et al. J. Neurosci. 28 (2008) 2959.

[8] J. Heymann et al., J. Struct. Biol. 166 (2009) 1.

[9] D. DeWinter et al., J. Microsc. 233 (2009) 372.

[10] R. Young et al., J. Microsc. 172 (1993) 81.

[11] H. Ngo et al., Am. J. Dentistry 13 (2000) 31D.

[12] V. Jantou et al., Micron 40 (2009) 495.

[13] K. Scott et al., J. Microsc. 233 (2009) 331.

[14] V. Raffa et al., Biomedical Microdevices 9 (2007) 371.

[15] E. Martinez et al., Micron 39 (2008) 111.

[16] H. Engqvist et al., J. Mater. Sci. 19 (2008) 467.

[17] H. Edwards et al., J. Microsc. 234 (2009) 16.

[18] J. Pelka et al., Chem. Res. Toxicology 22 (2009) 649.

[19] E. Schröder-Reiter et al., J. Struct. Biol. 165 (2009) 97.

[20] L. Hekking et al., J. Microsc. 235 (2009) 336.

[21] B. Lee et al., Microsc. Res. Techn. 70 (2007) 34.

[22] M. Marko et al., this Proceedings.

[23] Supported by NIH / NCRR Biomedical Research Technology Program Grant RR01219 (P.I . C.

Mannella). 